\title{
Ambiente institucional: construção e estudo exploratório de um questionário em contexto de ensino superior
}

\section{Institutional environment: design and exploratory study of a questionnaire in a higher education context}

\author{
Sofia de Lurdes Rosas da Silva*, Joaquim Armando Gomes Ferreira**, António Gomes Ferreira** \\ *Escola Superior de Educação - Instituto Politécnico de Coimbra, GRUPOEDE - CEIS XX, Portugal **Faculdade de Psicologia e de \\ Ciências da Educação da Universidade de Coimbra, GRUPOEDE - CEIS XX, Portugal
}

\begin{abstract}
Resumo
A literatura sobre ensino superior tem dado destaque ao estudo do ambiente institucional, indicando que as perceções dos estudantes acerca da qualidade dos ambientes institucionais apresentam impacto no seu ajustamento e desenvolvimento. Com este estudo pretendeu-se desenvolver um questionário para avaliar a perceção do estudante sobre o Ambiente Institucional (Questionário do Ambiente Institucional - QAI). Assim, partimos da análise de conteúdo de entrevistas realizadas a 31 estudantes e do seu confronto com a literatura. A versão final do QAI resultou numa solução de dois fatores, com níveis de consistência interna bastante aceitáveis: Estímulo Intelectual $(\alpha=.90)$, Sentimento de Comunidade $(\alpha=.88)$. Palavras chave: QAI, construção, estudo exploratório
\end{abstract}

\begin{abstract}
Literature on higher education has focused its attention on the study of institutional environment, pointing out that students' perceptions about the quality of institutional environments have an impact on their adjustment and development. The purpose of this study was to develop a questionnaire to evaluate students' perceptions about institutional environment (Institutional Environment Questionnaire - QAI). Thus, we started with the content analysis of interviews conducted with 31 students and its confrontation with literature. The final version of the QAI resulted in a two-factor solution with very acceptable levels of internal consistency: Intellectual Stimulus ( $\alpha=$ $.90)$, Sense of Community ( $\alpha=.88$ ).

Keywords: QAI, designing, exploratory study
\end{abstract}

\section{Introdução}

O ambiente institucional tem sido um constructo amplamente estudado nos contextos de ensino superior. Apesar de a literatura assumir a sua natureza multidimensional, uma parte substancial dos estudos tem-se situado sobretudo na análise do impacto das perceções dos estudantes em relação a diversas características institucionais. Este interesse resulta em parte pelo facto de as evidências empíricas apontarem para a forte ligação entre as perceções que se tem do ambiente institucional e o ajustamento, a aprendizagem e o desenvolvimento do estudante (Good \& Adams, 2008;
Kuh, Kinzie, Buckley, Bridges, \& Hayek, 2006; Laird \& Niskodé-Dossett, 2011; Lin, 2011; Meeuwise, Severiens, \& Born, 2010; Reason, Terenzini, \& Domingo, 2007; Silva, 2012; Silva, Ferreira, \& Ferreira, 2013, 2014; Wei, $\mathrm{Ku}, \&$ Liao, 2011). A literatura também se refere a um conjunto de práticas educativas institucionalmente pensadas e organizadas para promover o desenvolvimento e o ajustamento bem-sucedido do estudante. Acredita-se que um ambiente institucional resultará num impacto positivo se as práticas institucionais forem adequadas às características desenvolvimentais dos estudantes, em particular, se forem intelectualmente desafiantes e apoiantes (Kuh et al., 2006; Pascarella \& Terenzini, 2005).

As instituições de ensino superior apresentam um conjunto de tarefas académicas, de natureza intelectual e social, que exigem o envolvimento do estudante para a sua resolução. Porém, a sua resolução bem-sucedida necessita do apoio da instituição, providenciado através de um conjunto de programas e serviços de natureza curricular e extracurricular e de um clima relacional positivo caracterizado por interações significativas e apoiantes entre os elementos que compõem a comunidade académica.

Face a este enquadramento, o objetivo principal desta investigação foi o de construir o Questionário do Ambiente Institucional (QAI), considerando as características culturais e educativas de uma instituição do ensino superior politécnico português, e efetuar o estudo das suas qualidades métricas (dimensionalidade e consistência interna).

\section{Método}

Como referido anteriormente, o objetivo desta investigação consistiu na construção de um instrumento de avaliação do ambiente institucional (QAI) adaptado às características culturais da instituição escolhida para fazer o estudo. Para concretizar este objetivo efetuou-se uma entrevista a 31 estudantes de uma instituição do ensino superior politécnico sobre as características que consideravam apoiantes e estimulantes na instituição de ensino superior que frequentavam. 
Para a construção do instrumento partiu-se da análise de conteúdo das entrevistas e do confronto das categorias que foram emergindo dessa análise com os principais resultados da investigação no domínio.

A primeira versão do instrumento foi testada com um grupo de 45 estudantes e o estudo exploratório, após alguns ajustes ao QAI, foi conduzido com uma amostra de 576 estudantes.

Os pontos que se seguem referem-se aos procedimentos adotados e apresentam os estudos de validação utilizados.

\section{Sujeitos}

No pré-teste do QAI participaram 45 estudantes (7 do sexo masculino e 38 do feminino), a frequentar o $1 .^{\circ}$ $(n=24), 2 .^{\circ}(n=9)$ e $3 .^{\circ}$ ano $(n=12)$ de diversos cursos de formação inicial que incluíam cursos na área das Artes, Educação e Comunicação, com idades compreendidas entre os 17 e os 44 anos.

No estudo exploratório participaram 576 estudantes, do $1 .^{\circ}(\mathrm{n}=195), 2 .^{\circ}(\mathrm{n}=187)$ e $3 .^{\circ}$ ano $(\mathrm{n}=194)$. De salientar que a amostra, maioritariamente do sexo feminino $(\mathrm{n}=403 ; 70 \%)$, reflete as características do contexto de estudo. Os estudantes do sexo masculino são mais velhos em média um ano $(M=21.57 \pm 3.75)$ do que os do sexo feminino $(\mathrm{M}=20.59 \pm 2.80)$, diferença de idades considerada significativa $(\mathrm{t}=2.497 ; \mathrm{p}<.05)$.

\section{Procedimento}

Como referido, a construção do QAI partiu da análise de conteúdo das entrevistas realizadas aos estudantes e do confronto das categorias que foram emergindo dessa análise com os principais resultados da investigação no domínio. Da análise de conteúdo emergiram as seguintes categorias: Apoio Relacional, Integração das Necessidades, Estímulo Intelectual, Sentimento de Pertença, e Sentimento de Influência. O confronto das fontes levou-nos a partir do pressuposto da possibilidade de multidimensionalidade do constructo.

Para avaliar o constructo foram elaborados itens que, na sua maioria, emergiram do conteúdo das entrevistas realizadas. Procurou-se, nesta fase, elaborar-se o maior número de itens possível, para selecionar posteriormente os melhores itens a reter (Freire \& Almeida, 2001). Procedeu-se ainda a uma comparação com a literatura com o objetivo de refinar e completar o instrumento.

Neste processo também tiveram de ser tomadas decisões relativamente ao formato de resposta. A opção recaiu sobre a escala de likert de cinco pontos (totalmente em desacordo, bastante em desacordo, algumas vezes de acordo e outras em desacordo, bastante em acordo, totalmente em acordo).

Depois de elaborado, o QAI foi inicialmente submetido a um pré-teste junto de um grupo de 45 estudantes, com os seguintes objetivos: 1) avaliar o nível de compreensibilidade das questões; 2) detetar questões inúteis ou redundantes; 3) identificar perguntas relevantes que estivessem ausentes; e 4) avaliar a qualidade gráfica do instrumento. Durante a administração desta versão, procedeu-se a uma reflexão falada dos itens.
Elaborada a versão final do QAI, procedeu-se à sua administração junto de uma amostra de 576 estudantes com o objetivo de realizar um estudo exploratório que permitisse não apenas reduzir a extensão do instrumento, como também avaliar a sua dimensionalidade e respetiva consistência interna. Para o efeito, efetuaram-se análises fatoriais exploratórias (método de extração em componentes principais, rotação ortogonal varimax) e análises da consistência interna dos fatores através do alpha de Cronbach. No ponto que se segue, apresentam-se os resultados.

\section{Resultados}

A versão inicial do QAI era constituída por 51 itens distribuídos por 5 dimensões: Apoio Relacional, Integração das Necessidades, Estímulo Intelectual, Sentimento de Pertença, e Sentimento de Influência.

No que diz respeito à subescala Apoio Relacional, esta era constituída por 6 itens que se referem a uma dimensão relacional apoiante do ambiente institucional. Resultados elevados nesta subescala traduziriam perceções de um ambiente relacional apoiante e resultados baixos indicariam perceções de um ambiente relacional distante e "frio".

A subescala Integração das Necessidades integrava 12 itens que se centram nas provisões de suporte com vista à integração das necessidades educativas dos estudantes, tais como: oportunidades de participação, perceção de apoio, disponibilidade de recursos educativos, perceção das recompensas (aprendizagem e reconhecimento). Resultados elevados nesta subescala traduziam a perceção de uma instituição que procura responder às necessidades educativas dos estudantes, enquanto resultados baixos indicariam perceções de uma instituição que não integra as necessidades dos estudantes nos serviços que presta.

No que diz respeito à subescala Estímulo Intelectual, os 17 itens que a constituíam avaliavam a perceção de estímulo intelectual através de aspetos como: oportunidades e incentivo à participação em atividades extracurriculares e curriculares, sentimento global de estímulo para aprender, perceções sobre o caráter desafiante em relação ao curso e à gestão pedagógica. Resultados elevados nesta subescala traduziriam a perceção de um ambiente institucional intelectualmente desafiante e estimulante.

No que concerne ao Sentimento de Pertença, os 11 itens que constituíam esta subescala referiam-se à perceção de que se é um membro da instituição, aceite pelos outros membros. O sentimento de pertença e aceitação incluía também itens que se referem à segurança emocional e aos laços de amizade. Resultados elevados nesta subescala traduziriam o sentimento de pertença e de integração na instituição.

Por fim, a subescala Sentimento de Influência era constituída por 6 itens que se referiam à perceção de que se detém algum poder para contribuir para o bem comum ou melhoria do funcionamento institucional. Resultados elevados nesta subescala traduziriam perceções de um ambiente que está aberto ao contributo dos estudantes 
para a melhoria das condições de funcionamento da instituição.

Numa primeira fase do estudo, para os 51 itens que avaliavam o Ambiente Institucional, os indicadores fornecidos pelo teste KMO [Kaiser-Meyer-Olkin Measure of Sampling Adequacy =.955], pelo teste de esfericidade de Bartlet [ $\chi 2(1275)=14779,737, \mathrm{p}<.001]$ e pela matriz anti-imagem (com valores na diagonal principal a oscilar entre .87 e .97$)$ revelaram-se adequados à prossecução da análise fatorial.

Os estudos preliminares com uma análise fatorial em componentes principais, rotação ortogonal varimax, revelaram 9 fatores com eigenvalues superiores a 1 , de difícil interpretação. A solução forçada a 2 fatores foi a que se revelou mais interpretável apesar da observação do gráfico de progressão dos valores próprios (scree test) sugerir a configuração de 1 fator. A solução forçada a 2 fatores revelou a necessidade de redução e reorganização do instrumento. Relembra-se aqui que numa fase inicial foram formulados o maior número de itens possível, para posteriormente se reterem os melhores (Freire \& Almeida, 2001).

No processo de redução e reorganização dos dados foram realizados sucessivos ensaios, tendo sido considerados os seguintes critérios (Hair, Anderson, Tatham, \& Black, 2006): i) critério de Kaiser (com valor próprio superior a 1); ii) peso fatorial dos itens igual ou superior a .30; iii) consistência interna, medida pelo alpha de Cronbach, superior a .70; iv) valor de comunalidades superior a .30; v) ponderação dos critérios estatísticos em função do valor conceptual do item para o constructo.

Face a estes critérios foram eliminados os seguintes itens: valor das comunalidades abaixo de .30 (itens 1,2 , $4,8,11,12,13,17,21,22,25,38,43)$; valor de saturação no fator abaixo de .30 (item 11); itens considerados inadequados do ponto de vista conceptual (itens 5, 16, $18,20,24,27,29,30,31,32,44,49)$, considerando o fator onde saturaram; itens com saturações elevadas em mais que um fator (itens 10, 26, 28, 41, 46, 51. Considerou-se como limite de corte saturações cuja diferença de saturação $\leq .1$, uma vez que a partir deste valor se considera que o item apresenta pouco ou nenhum poder discriminativo.

Foi realizada a Análise Fatorial em Componentes Principais, rotação ortogonal varimax, com o conjunto dos 20 itens. Os indicadores fornecidos pelo teste KMO $[K M O=.940]$ e pelo teste de esfericidade de Bartlet $[\chi 2$ $(190)=5421,636, p<.001]$ indicaram a viabilidade da análise fatorial, o mesmo acontecendo com a matriz anti imagem com valores na diagonal principal que oscilaram entre .91 e .97 .

A solução encontrada evidenciou uma estrutura fatorial organizada em dois fatores que em conjunto explicam 50.7 \% da variância. Após a rotação, o fator 1 apresenta um valor próprio de 5.28 e explica $26,4 \%$ da variância e o fator 2 tem um valor de 4.97 e explica $24.7 \%$ da variância (cf. Quadro 1). Os valores das comunalidades $\left(\mathrm{h}^{2}\right)$ registadas nos 20 itens variaram entre $.39<\mathrm{h}^{2}<.63$.

Quadro 1
Análise Fatorial dos 20 itens do QAI

\begin{tabular}{|c|c|c|c|}
\hline \multirow{2}{*}{ Itens } & \multicolumn{2}{|c|}{ Fatores } & \multirow[b]{2}{*}{$\mathbf{h}^{2}$} \\
\hline & 1 & 2 & \\
\hline $\begin{array}{l}\text { 50. As aulas são muito importantes } \\
\text { para a minha aprendizagem. }\end{array}$ & .77 & .18 & .63 \\
\hline $\begin{array}{l}\text { 36. O meu curso tem-me proporcionado } \\
\text { uma visão alargada da minha área de } \\
\text { formação. }\end{array}$ & .77 & .17 & .62 \\
\hline $\begin{array}{l}\text { 37. O curso leva-me a procurar novas } \\
\text { ideias e conhecimentos. }\end{array}$ & .74 & .20 & .60 \\
\hline $\begin{array}{l}\text { 34. Sinto que tenho realizado } \\
\text { aprendizagens a nível pessoal e social }\end{array}$ & .72 & .23 & .58 \\
\hline $\begin{array}{l}\text { 42. Considero as disciplinas do meu } \\
\text { curso, intelectualmente estimulantes. }\end{array}$ & .67 & .25 & .52 \\
\hline $\begin{array}{l}\text { 47. O modo como são dadas as aulas } \\
\text { estimula-me a aprender. }\end{array}$ & .66 & .33 & .55 \\
\hline $\begin{array}{l}\text { 35. Nesta instituição sempre exigiram } \\
\text { o meu melhor. }\end{array}$ & .65 & .30 & .52 \\
\hline $\begin{array}{l}\text { 48. Os materiais de apoio que me são } \\
\text { disponibilizados são relevantes e } \\
\text { atualizados. }\end{array}$ & .62 & .27 & .46 \\
\hline $\begin{array}{l}\text { 33. Na minha instituição tenho acesso } \\
\text { aos materiais e recursos necessários ao } \\
\text { meu curso. }\end{array}$ & .54 & .39 & .44 \\
\hline $\begin{array}{l}\text { 19. Aqui somos estimulados a } \\
\text { estabelecer contactos com as outras } \\
\text { pessoas dentro e fora da instituição. }\end{array}$ & .50 & .37 & .39 \\
\hline
\end{tabular}

Fator 1: Estímulo Intelectual; Valor próprio $=5.28$; Variância explicada $=26.4 \%$

39. Sinto que, nesta instituição, as $\begin{array}{llll}\text { opiniões e sugestões dos estudantes são } & .22 & \mathbf{. 7 0} & .55\end{array}$ tidas em conta.

$\begin{array}{lllll}\text { 6. Nesta instituição sinto-me em casa. } & .21 & \mathbf{. 6 9} & .52 \\ \text { 9. A instituição apoia-me naquilo que } & & & \end{array}$ preciso.

$\begin{array}{lll}.21 & .67 & .49\end{array}$

14. Nesta instituição, os diferentes órgãos estão abertos à discussão dos problemas que nos afetam.

7. Nesta instituição sinto que posso dar o meu contributo para melhorar o seu funcionamento.

40. Estou satisfeito(a) com o ambiente vivido nesta instituição.

15. Nesta instituição sente-se que há um relacionamento de proximidade e apoio entre os estudantes.

3. Sinto que quando os estudantes têm uma iniciativa, há apoio por parte da instituição.

45. Dentro da instituição, se eu o desejar, são-me dadas oportunidades para dar o meu contributo.

$.20 \quad .65 \quad .46$

23. Nesta instituição sinto que posso contar com as outras pessoas (professores, estudantes, funcionários).

Fator 2: Sentimento de Comunidade; Valor próprio $=4.97$; Variância explicada $=24.7 \%$

O fator 1 é constituído por 10 itens e apresenta saturações que variam entre .50 e .77 . Os itens que o compõem pertenciam originalmente à subescala Estímulo Intelectual com exceção dos itens 33 e 34 (Integração das Necessidades). Uma análise conceptual 
destes itens conduziu à reconsideração da sua posição original, passando estes a integrar o fator 1 que se continuou a designar de Estímulo Intelectual. Note-se que os itens 47, 33 e 19 apresentam valores de saturação $>.30$ no segundo fator. Apesar de Nunnally e Bernstein (1994) defenderem que quando os itens saturam em mais que um fator com valores acima de .30 se deva eliminá-los, considerando que se trata ainda de um estudo exploratório, nesta fase optou-se por mantê-los, salientando, porém, a necessidade de mais estudos com outras populações.

O fator 2 integra 10 itens com saturações que oscilam entre .55 e .70 . Este fator congrega itens que originalmente constituíam as subescalas Integração das Necessidades, Apoio Relacional, Sentimento de Pertença e Sentimento de Influência (que teoricamente se constituem nas dimensões do sentimento de comunidade). A análise conceptual dos itens conduziu à decisão de reorganizar os itens que avaliavam diferentes dimensões do sentimento de comunidade numa só dimensão que se passou a designar Sentimento de Comunidade.

Para estudar a fidelidade das dimensões do Ambiente Institucional procedeu-se ao cálculo do coeficiente alpha de Cronbach ( $\alpha$ ) para cada dimensão e, para cada item, calculou-se a média, o desvio-padrão e as correlações entre cada item e o total da dimensão correspondente. Uma análise dos itens permite verificar que a generalidade se correlaciona com a escala total em valores superiores a .30, valor mínimo a reter segundo Cronbach (1984) (cf. Quadro 2). Os valores totais obtidos revelaram-se bastante razoáveis: Estímulo Intelectual $(\alpha=.90)$; Sentimento de Comunidade $(\alpha=$ $.88)$.

Quadro 2.

Consistência interna (alpha de Cronbach) do QAI

\begin{tabular}{|c|c|c|c|c|}
\hline Item & Média & $\begin{array}{l}\text { Desvio- } \\
\text { Padrão }\end{array}$ & $\begin{array}{l}\text { Correlação } \\
\text { Item-total }\end{array}$ & $\begin{array}{c}\alpha \text { com } \\
\text { exclusão } \\
\text { do item }\end{array}$ \\
\hline 19 & 3.34 & .86 & .55 & .89 \\
\hline 33 & 3.28 & .93 & .59 & .89 \\
\hline 34 & 3.79 & .74 & .68 & .88 \\
\hline 35 & 3.45 & .83 & .65 & .88 \\
\hline 36 & 3.70 & .89 & .69 & .88 \\
\hline 37 & 3.84 & .80 & .68 & .88 \\
\hline 42 & 3.19 & .90 & .64 & .88 \\
\hline 47 & 3.13 & .80 & .67 & .88 \\
\hline 48 & 3.52 & .81 & .61 & .89 \\
\hline 50 & 3.81 & .84 & .69 & .88 \\
\hline \multicolumn{5}{|c|}{ Estímulo Intelectual $\alpha=.90$} \\
\hline 3 & 3.29 & .82 & .55 & .87 \\
\hline 6 & 3.13 & .94 & .63 & .86 \\
\hline 7 & 3.06 & .86 & .60 & .86 \\
\hline 9 & 2.97 & .84 & .61 & .86 \\
\hline 14 & 3.06 & .89 & .58 & .87 \\
\hline 15 & 3.50 & .83 & .58 & .87 \\
\hline 23 & 3.52 & .75 & .57 & .87 \\
\hline 39 & 3.19 & .86 & .65 & .86 \\
\hline 40 & 3.51 & .85 & .68 & .86 \\
\hline 45 & 3.35 & .74 & .59 & .87 \\
\hline
\end{tabular}

Sentimento de Comunidade $\alpha=.88$
O primeiro fator designado por Estímulo Intelectual integra itens que avaliam a perceção de estímulo institucional para o desenvolvimento intelectual, académico e pessoal do estudante. Os itens abordam aspetos como: incentivo à participação em atividades curriculares, sentimento global de estímulo para aprender, perceções sobre o caráter desafiante em relação ao curso e gestão pedagógica, disponibilidade de recursos educativos, perceção de crescimento pessoal e social.

O fator Sentimento de Comunidade engloba os itens relativos às quatro dimensões do sentimento de comunidade: sentimento de pertença, sentimento de influência, satisfação e integração de necessidades e laços emocionais.

\section{Discussão}

Neste artigo apresentam-se os procedimentos adotados para a construção do QAI e os dados métricos do estudo exploratório. Efetuou-se uma Análise Fatorial Exploratória para averiguar as componentes principais do QAI. Apesar do pressuposto inicial de multidimensionalidade, a solução fatorial encontrada de dois fatores, que explica 50,7\% da variância, pareceu-nos com sentido conceptual.

Considerando a literatura (Hair et al., 2006), o facto de termos encontrado correlações item-total do fator superiores a .50 para todos os itens é um indicador de existência de níveis de consistência interna bastante aceitáveis.

No que concerne aos resultados da comunalidades todos os itens atingem valores acima de .30

Quanto aos pesos dos itens que constituem o fator, verificamos que todos os itens apresentam pesos fatoriais aceitáveis (superiores a .50) (Hair et al., 2006).

$\mathrm{O}$ valor do alpha de Cronbach encontrado para ambos os fatores traduz uma muito boa consistência interna (Hill \& Hill, 2009).

Os resultados obtidos neste estudo exploratório são reveladores das boas qualidades métricas deste instrumento.

Concluímos pela necessidade de mais estudos que atestem as qualidades métricas do Questionário do Ambiente Institucional, esperando que este se constitua não apenas num instrumento útil em contexto de investigação, mas sobretudo como um instrumento de diagnóstico do ambiente institucional, permitindo identificar situações que necessitem de reflexão e de intervenção institucional.

\section{Referências}

Cronbach, L. (1984). Essentials of psychological testing ( $4^{\text {th }}$ ed.). New York: Harper \& Row.

Freire, T., \& Almeida, L. (2001). Escalas de avaliação, construção e validação. In E. Fernandes \& L. Almeida (Eds.), Métodos e técnicas de avaliação: Contributos para a prática e investigação psicológicas (pp. 109-128). Braga: Universidade do Minho, Centro de Estudos em Educação e Psicologia. 
Good, M., \& Adams, G. (2008). Linking academic social environments, ego-identity formation, ego virtues, and academic success. Adolescence, 43 (170), 221-236.

Hair, J., Anderson, R., Tatham, R., \& Black, W. (2006). Multivariate data analysis ( $6^{\text {th }}$ ed.). New Jersey: Pearson Education, Inc.

Hill, M., \& Hill, A. (2009). Investigação por questionário. Lisboa: Edições Sílabo.

Kuh, G., Kinzie, J., Buckley, J., Bridges, B., \& Hayek, J. (2006). What matters to student success: a review of the literature. Commissioned report for the national symposium on postsecondary student success: Spearheading a dialog on student success. Symposium conducted at the meeting of the National Postsecondary Education Cooperative, Washington, DC. Retrieved from http://nces.ed.gov/npec/pdf/kuh_team_report.pdf

Laird, T., \& Niskodé-Dossett, A. (2010). How gender and race moderate the effect of interactions across difference on student perceptions of the campus environment. The Review of Higher Education, 33 (3), 333-356.

Lin, Y. (2011). University environment experience of the first two years of university graduates at the newly established small university located in suburban area in Taiwan. College Student Journal, 45 (1), 65-83.

Meeuwisse, M., Severiens, S., \& Born, M. (2010). Learning environment, interaction, sense of belonging and study success in ethnically diverse student groups. Research in Higher Education, (51), 528-545.

Nunnally, J., \& Bernstein, I. (1994). Psychometric theory (3rd ed.). New York: MacGraw-Hill.

Pascarella, E., \& Terenzini, P. (2005). How college affects students: a third decade of research. San Francisco: Jossey-Bass.

Reason, R., Terenzini, P., \& Domingo, R. (2007). Developing social and personal competence in the first year of college. The Review of Higher Education, 30 (3), 271-299.

Silva, S. (2012). Dinâmicas de envolvimento e de desenvolvimento do estudante do ensino superior. Tese de Doutoramento apresentada à Faculdade de Psicologia e de Ciências da Educação da Universidade de Coimbra, Coimbra, Portugal.

Silva, S., Ferreira, J. A., \& Ferreira, A. (2013). Vivências académicas e sociais e perceções de desenvolvimento: Dados de um estudo com estudantes do ensino superior politécnico. In B. D. da Silva, L. S. Almeida, A. B. Lozano, M. P. Uzquiano, A. Franco, \& R. Monginho (Orgs.), Atas do XII Congresso Internacional Galego-Português de Psicopedagogia (pp.7243-7258). Braga: Universidade do Minho.

Silva, S., Ferreira, J. A., \& Ferreira, A. (2014). Vivências no ensino superior e percepções de desenvolvimento: Dados de um estudo com estudantes do ensino superior. Revista E-Psi - Revista Eletrónica de Psicologia, Educação e Saúde, 4 (1), 5-27. Disponível em http://www.revistaepsi.com

Wei, M., Ku, T., \& Liao, K. (2011). Minority stress and college persistence attitudes among African American, Asian American, and Latino students: Perception of university environment as a mediator. Cultural
Diversity and Ethnic Minority Psychology, 17 (2), 195-203. 